\title{
Germanica
}

\section{Une saison en Galicie : autopsie d'une langue}

À propos de Die Galizianerin de Brigitte Schwaiger et Eva Deutsch

Eine Zeit in Galizien: Autopsie einer Sprache. Über Die Galizianerin von Brigitte

Schwaiger und Eva Deutsch

\section{Astrid Starck}

\section{OpenEdition}

\section{Journals}

\section{Édition électronique}

URL : http://journals.openedition.org/germanica/2623

DOI : 10.4000/germanica.2623

ISSN : $2107-0784$

\section{Éditeur}

Université de Lille

\section{Édition imprimée}

Date de publication : 31 décembre 1989

Pagination : 41-53

ISSN : 0984-2632

\section{Référence électronique}

Astrid Starck, «Une saison en Galicie : autopsie d'une langue », Germanica [En ligne], 5 | 1989, mis en ligne le 09 février 2015, consulté le 06 octobre 2020. URL : http://journals.openedition.org/germanica/ 2623 ; DOI : https://doi.org/10.4000/germanica.2623

Ce document a été généré automatiquement le 6 octobre 2020.

(c) Tous droits réservés 


\title{
Une saison en Galicie : autopsie d'une langue
}

\author{
À propos de Die Galizianerin de Brigitte Schwaiger et Eva Deutsch \\ Eine Zeit in Galizien: Autopsie einer Sprache. Über Die Galizianerin von Brigitte \\ Schwaiger und Eva Deutsch
}

Astrid Starck

1 Die Galizianerin (La Galicienne) est une œuvre «bicéphale » qui parut à Vienne en 1982 aux éditions Zsolnay. «Bicéphale », car les auteurs en sont Brigitte Schwaiger, écrivain, et Eva Deutsch, témoin. Le livre repose en effet sur le témoignage oral d'une femme juive de Galicie - c'est là le sens du mot allemand Galizianerin - née à Cieszanow, près de Przemysl - où passait la ligne de partage linguistique entre le polonais et l'allemand, et entre le polonais et l'ukrainien -, en 1924, à une époque où cette province venait de cesser de faire partie de la Monarchie austro-hongroise pour retourner à la Pologne. Néanmoins, du point de vue de son histoire personnelle, la narratrice se considère comme Galicienne (page 11). Dans la production littéraire de l'auteur, cette œuvre semble à première vue occuper une place à part. Sans doute est-ce le cas en ce qui concerne la langue dans laquelle elle est écrite: elle constitue la tentative de reproduire, à l'intérieur de l'écriture, un langage oral particulier, un langage déstructuré qui devient le miroir d'un univers éclaté et, en dernière analyse, de l'absence de langage et d'identité de la femme.

2 Mais ce livre semble annoncé déjà dans Lange Abwesenheit (Une aussi longue absence), qui parut en 1980 aux mêmes Éditions, où la narratrice, Je, manifeste son désir d'être confrontée au passé récent (page 51). Cette œuvre s'insère à la fois dans la thématique schwaigerienne - la recherche d'une identité féminine - et dans sa problématique - la recherche d'une identité après la guerre et la confrontation avec les événements récents, à savoir l'antisémitisme et la "Shoah». Si Ilse Aichinger a vécu la guerre et l'exclusion raciale (Die größere Hoffnung, 1948 - Le grand espoir, 1956), si Ingeborg Bachmann avait assisté à l'entrée des troupes de Hitler à Klagenfurt, ce qu'elle ne put jamais oublier («Jugend in einer österreichischen Stadt», in : Das dreißigste Jahr, 1961 - 
"Jeunesse dans une ville autrichienne ", in La trentième année, 1964), Brigitte Schwaiger, née en 1949 , fait partie de la génération d'après-guerre qui découvre le passé nazi. Ingeborg bachmann avait identifié le sort de la femme au destin du peuple juif en faisant endosser à l'héroïne de Malina (1971) le manteau juif de Sibérie.

Dans cette société où la femme est assassinée psychologiquement, les meurtriers d'antan sont toujours actifs :

Je me suis souvent demandé, dit Franza, et vous aussi, où était passé le virus du crime. Il ne peut tout de même pas avoir disparu d'un seul coup de notre univers il y a une vingtaine d'années, simplement parce que le meurtre n'est plus de nos jours mis en valeur, exigé, récompensé par des médailles et encouragé. Certes, les massacres sont du passé, les assassins eux sont encore parmi nous... On nous fait prendre conscience de l'existence de ces assassins, non par le biais de reportages plus ou moins pudiques, mais bien plutôt par la littérature' ${ }^{1}$.

4 La recherche de l'identité féminine va de pair avec une réflexion sur la société. Il est intéressant de noter que le thème abordé par Brigitte Schwaiger l'est sous l'angle de la femme : son rôle de courroie de transmission d'un passé projeté en elle par la famille et la religion l'oblige à opérer une rupture brutale, à se situer à chaque instant pour sortir de son néant d'être et devenir ainsi une identité en train de se créer. Mais cette recherche ne doit pas être perçue du seul point de vue de la femme: elle devrait entraîner une réflexion de l'homme sur son identité massive. Cette tentative féminine est une possibilité pour lui de se redéfinir ${ }^{2}$. Cette recherche s'opère sur la base d'une double démarche : d'une part, en se soustrayant à une idéologie qui la veut objet tradition et religion - et dont le véhicule est l'écriture, d'autre part en faisant surgir un autre passé qui se délivre à travers une parole qui tente de le dire.

Dans Der Himmel ist süß, «Eine Beichte» (Le ciel est suave, Une confession), de 1984, le trio grand-mère, mère, fille illustre la transmission inexorable orchestrée par le père, regard omniscient. C'est à propos de la révolte contre le père, porteur de tous les clichés religieux, sociaux et raciaux que va surgir la confrontation avec l'antisémitisme. À Je/narrateur dans Lange Abwesenheit, réceptacle des idées du père («Manchmal ist mir, als trüge ich anstelle des Kopfes ein Blechgeschirr mit zwei Henkeln, in das jeder seinen Dreck hineinschütten kann» - "Parfois j'ai l'impression d'avoir en guise de tête un récipient en fer-blanc à deux anses où chacun peut déverser ses ordures ", page 31), se superpose Je/actant, maîtresse de Birer, Juif de la génération de son père, incarnation de l'altérité («Wenn in meinem Hirn ein Kabel steckte, das ich an sein Hirn anstecken könnte!» - «S'il pouvait y avoir un câble dans mon cerveau que je puisse raccorder au sien !", page 61). Ces deux connexions de Je- le père et Birer- l'installent dans un chassécroisé. Dans cette histoire triangulaire où Je va de l'un à l'autre, elle se pose comme le regard intérieur et extérieur, mais elle est aussi la balle que les deux protagonistes se renvoient. Birer, soumis au regard du père/fille, peut être vu comme le véhicule d'une image qui l'installe dans un rôle historique négatif. En cela il fonctionne comme le double de Je qui se mire dans cette absence d'identité, ou dans cette identité préétablie. Echapper à cette image, c'est instaurer une identité propre : la jeunesse de Je/maîtresse en serait la métaphore. Birer devient bien, dans sa réalité phénoménologique, le poseur de questions muettes, il porte témoignage, il devient le regard autre : le sien et celui de tous ceux qui ont disparu. Mais il ne peut faire éclater le carcan qui l'emprisonne. Il ne peut créer de langage nouveau. Il empêche Je de parler.

6 Ce passage à la parole sera tenté dans Die Galizianerin qui deviendra ainsi l'expression de l'altérité : celle d'une femme juive. Cette œuvre s'insère à la fois dans la thématique 
schwaigerienne - la révolte d'une femme contre la tradition - et dans sa problématique - la dénonciation de l'agression des autres allant jusqu'à l'extermination -. Contrairement à Birer qui est de la même génération qu'elle, elle libère une voix vivante qui témoigne.

7 Par son témoignage, elle renoue avec la mémoire collective qui l'insère dans un avant, mais qui ne lui permet pas de se structurer dans un après. Car sa voix de femme continue à être brisée dans un aujourd'hui, reflet du passé. Ses origines de Galicienne ne relèvent pas d'une province mythique, mais d'un anéantissement historique, transcendé en dernier ressort dans un univers imaginaire, ni juif, ni autrichien, ni polonais qui s'exprimerait à travers une "interlangue ", ni yidich, ni allemande, permettant la transition vers la cosmopolis. Le témoignage se délivre comme une interpellation directe de l'interlocutrice ignorante - Savez-vous? (Wissen Sie?) scande toute la narration - et muette - elle n'intervient jamais -, et derrière elle, de la lectrice (et du lecteur) auquel le livre s'adresse. Il apparaît comme un questionnement sans relâche auquel succède l'absence de réponse. Dans ce pan d'histoire vécue relatée oralement, le dire est déjà le faire. Une femme parvient à échapper à l'extermination nazie après avoir perdu tous les siens. Eva Deutsch, la Galicienne, met en scène Chawa Frankel - son double adolescent - en l'éclairant rétrospectivement à la lumière des événements récents en Autriche. En effet, le livre s'ouvre sur l'antisémitisme dans l'Autriche d'aujourd'hui. Si bien que cette vie relatée, tout en étant une réminiscence, devient également un présent qui perdure. En ce sens, il rejoint la problématique de Lange Abwesenheit.

8 Cette problématique trouve une nouvelle expression dans le recueil de poèmes Mit einem möcht' ich leben (Je voudrais vivre avec un gars), paru en $1987^{3}$. Le long poème en prose, sorte de ballade brechtienne, intitulé «Führer befiehl» (« Führer, ordonne ») met en scène une adolescente nazie pendant et après la guerre que la psychologie de masse $d u$ fascisme $^{4}$ satisfait sur le plan de l'éducation, de la religion, de la tradition et de l'identité («Und du bist so viel wert wie ein Mann» - «Et tu vaux tout autant qu'un homme», page 71). Cette femme qui rêvait de devenir Führerin («Führer» au féminin) se voit déclassée dans la hiérarchie aryenne du fait de ses origines juives; mais elle ne perd rien de son ardeur après la guerre. Au contraire. La femme apparaît comme le réceptacle du délire de l'homme et tout accès à une identité propre lui est définitivement barré.

9 Ces trois œuvres à la première personne qui forment en quelque sorte un triptyque mettent en lumière la thématique et la problématique de Brigitte Schwaiger. Partant d'une situation actuelle, elles opèrent, dans une langue qui court-circuite la logique du discours, la perpétration de clichés qui deviennent, dans leur littéralité, la manifestation de l'extériorisation masculine. Ce procédé qui ne permet pas d'esthétisme accentue le caractère masculin de l'expression de la femme, de son absence de langage propre et, par conséquent, de son absence d'identité. L'auteur juxtapose les clichés et ce qui les réfute: des idées et des actions contradictoires se trouvent ainsi confrontées les unes aux autres, sans articulation aucune. C'est dans cet interstice muet que se glisse la rupture et l'ébauche d'une expression féminine. Si dans l'écriture, les blancs avant et après le point peuvent constituer un début de balbutiement, le récit dans Die Galizianerin utilise des raccourcis éloquents où la parole devient synonyme d'action. N'est-ce pas au vu de ses déclarations verbales dénonçant la guerre et l'antisémitisme que la narratrice risque des poursuites judiciaires (page 7 
sq.) ? Si l'écriture a besoin d'être court-circuitée pour signifier, la parole immédiate est, dans sa corporéité, du sens fait chair. C'est pourquoi, des trois volets du triptyque, Die Galizianerin est le seul à constituer une mise en dire qui est une mise/remise en vie : voilà le rôle du survivant qui témoigne («Sehen Sie, hat immer Gott geschickt, daß jemand ist zurückgeblieben. Hat der Christ das [jüdische] Mädel herausgenommen, und dieses Mädel war das Wunder, daß sie soll Zeuge sein, was man gemacht hat» « Voyez-vous, Dieu a toujours fait en sorte qu'il y ait un survivant. Le Chrétien a déterré la fillette [on venait de l'enterrer vive, note de l'auteur] et cette fillette, voilà le miracle, c'est elle qui témoignerait », page 44).

10 La Galicie, province juive, devient le condensé de différents contenus qui se superposent et se complètent et sa pluralité même confère au texte sa multidimensionalité. Si le titre du livre renvoie à une province d'origine, la langue renvoie à un univers déstructuré. C'est la voix de la mémoire collective - « Nous autres, Juifs» («Wir Juden», page 12) - qui tente de se faire entendre. Il ne s'agit plus, comme dans Die Wasserträger Gottes (Porteurs d'eau) de Manès Sperber ${ }^{5}$, d'un monde organisé réorganisé après-coup - qui pourrait laisser au lecteur l'illusion, un instant, d'une existence qui n'aurait pas été mise en cause ; il ne s'agit pas non plus, comme dans Hiob. «Roman eines einfachen Mannes» (Job, «Roman d'un simple Juif») de Joseph Roth ${ }^{6}$, d'une vision nostalgique d'un monde après la destruction qui fit suite à la première guerre mondiale. Non: le lecteur assiste, impuissant, à la destruction systématique d'une région et à l'extermination de sa population ${ }^{7}$, relatée par la narratrice qui opère des incursions dans le passé. Celui-ci prend un aspect ambigu, dans la mesure où l'accent est mis sur ce qu'il a de contraignant pour une jeune fille qui aimerait s'émanciper de la tutelle exercée par la tradition et la religion. Mais en même temps pèse sur ce récit la disparition de tout un monde, et cette disparition lui confère un caractère qui transcende ce problème spécifique. Ce que Joseph Roth voyait dans la Galicie, ce que Kafka avait découvert dans le judaïsme d'Europe Centrale, c'était l'expression d'une judéité parcourant toutes les manifestations de la vie. Cette appartenance et cet attachement à la judéité devient la raison de vivre d'Eva Deutsch/ narratrice ; elle insiste sur ce point malade, elle ne veut pas mourir, chrétienne, sous le nom de Jadviga Godovska (page 217) ; après la guerre, installée à Presbourg/Bratislava, elle ne veut pas abjurer sa religion comme le lui conseille son mari (page 112). « Suivant dans nos sales brouillards des shtelekh absents les fantômes épars ${ }^{8}$ ", elle témoigne pour ce monde qui n'existe plus. Le shtetl, entité historique, économique, religieuse et culturelle était l'humus de la judéité. Mais déjà en 1914, il était une réalité imaginaire en pleine déconstruction, réalité qui, à partir de la seconde moitié du $\mathrm{XX}^{\mathrm{e}}$ siècle, est devenue une "perspective». Les incursions dans le passé contenues dans Die Galizianerin mettent en lumière la vie communautaire d'Europe Centrale. Elles ponctuent le récit comme une accalmie douloureuse, mais aucune évocation ne peut être menée à terme : à tout moment, la guerre fait irruption.

11 Si l'œuvre s'insère dans une préoccupation récente en Autriche (les années 80 voient fleurir toute une littérature sur le sujet - anthologie de textes ${ }^{9}$, recueil d'essais ${ }^{10}$, roman $^{11}$, film ${ }^{12}$ et colloque ${ }^{13}$-, c'est parce que la Galicie devient une métaphore : celle de la monarchie disparue - Habsburgische Atlantis ${ }^{14}$-, mais aussi celle de la "Shoah». Elle relève à la fois du mythe ${ }^{15}$ - l'âge d'or d'un judaïsme religieux et séculier - et de la destruction - Galizien als Bedrohung ${ }^{16}$. Pourtant, à l'époque où elle existait encore, la Galicie était déjà un microcosme dans le macrocosme que représentait la Monarchie : creuset de multiples nationalités, religions, coutumes et langues, elle se présentait dans 
sa diversité baroque et constituait le reflet de cette Monarchie décadente, porteuse de mort, de cette fin-de-siècle morbide et irréversible ${ }^{17}$.

Dans Die Galizianerin, la description de cette province qui revient comme un leitmotiv est marquée d'un double sceau : celui de l'ébranlement de la tradition et celui de la disparition. Les évocations du passé reposent sur des clichés (Kaftan, Barte, Peies, p. 12 sq.) qui fonctionnent comme des instantanés, vestiges ou souvenirs ${ }^{18}$. En d'autres termes, ce monde qui était le berceau du Hassidisme et de la Haskalah, de la mystique et des Lumières juives, de deux mouvements opposés où s'affrontaient tradition et modernité, ne peut plus se dire qu'à travers une reconstruction sporadique et fragmentaire, soumise au regard de la narratrice / femme (ses aspirations se dévoilent comme étant réservées aux seuls hommes).

$13 \mathrm{Au}$ télescopage de ces deux mondes se superpose le rôle et l'impact de la culture allemande telle qu'elle est perçue dans cet avant-poste de la germanité : longtemps symbole d'humanisme, de modernité et d'ouverture sur l'Occident (page 18 sq.), elle se mue en son contraire, la barbarie (page 19). D'une manière fulgurante, cette antinomie se fait jour en raccourci : les soldats allemands, porteurs de culture («Die deutschen Soldaten waren so kultiviert, so nobel», page 10), ne sont plus que des assassins («Nur morden hat er [der Deutsche] wollen», page 13). Là se manifeste toute la dérision. Cette idée de culture allemande dans cette province pour laquelle Schiller représentait l'écrivain par excellence ${ }^{19}$ était si profondément ancrée que, pendant longtemps, on ne put croire à la réalité des événements.

Du fait du télescopage du monde de la tradition et de la modernité, de celui de la culture allemande et de la barbarie, Die Galizianerin ne peut laisser s'installer une image de la Galicie qui serait a-historique :

Si les shtelekh existaient encore, ils n'appartiendraient pour moi qu'à un passé lointain ; mais comme on les a détruits et anéantis au point que rien de ce qu'ils ont été et auraient pu être ne peut trouver de prolongement dans l'avenir, Zablotow fait désormais partie de mon présent. Son lieu géographique, c'est ma mémoire ${ }^{20}$.

La narratrice a vécu la destruction: elle sait que "la mort est un maître venu d'Allemagne ${ }^{21} »$. La Galicie est tombée dans le silence.

Si le dire sur la Galicie ne peut aboutir, c'est qu'il est le reflet de cette alchimie $d u$ silence $^{22}$. Tout au plus peut-il s'écrire comme mythe: la parabole de «la branche cassée ", insérée dans le texte oral sous forme de lettre reprend l'histoire de la Genèse, de l'Exil et de l'errance. Cette mise en écriture y figure comme une " mise en abyme ${ }^{23}$ " qui condenserait le récit oral en lui conférant une organisation de récit structuré. Ce très beau texte, écrit en allemand, intègre la narratrice à un pays d'origine, la Galicie, qui aurait remplacé la terre des ancêtres dont on se souvient encore («les Juifs sont, dans cette province, des arbres exotiques qui ont pris racine", page 50 ; ils font désormais partie du paysage) ; c'est un havre de paix champêtre et d'harmonie. Mais un peuple étranger fait irruption qui veut exterminer cette population exotique. Devant ce massacre, Dieu se met à pleurer et transplante les arbres abattus au paradis afin qu'ils l'embellissent. Cependant, une branche se révolte contre son sort, se détache du tronc et s'en va. Consciente du danger et de son infinie faiblesse, elle se transforme et se métamorphose, parvenant ainsi à masquer ses origines exotiques tout en les préservant. Pour qu'elle ne succombe pas au milieu des étrangers, Dieu lui prête vie et pérégrination jusqu'à ce que vienne son heure. Cette parabole est suivie d'un chant en yidich (page 51 sq.) qui relate le retour en Galicie, la disparition des siens et l'absurdité 
de l'existence. La lettre se termine par une action de grâce à Dieu qui lui a permis de survivre pour que renaisse la vie (page 52). Dans ce passage à l'écriture, l'héroïne perd le caractère hybride qu'elle a dans le récit oral, mais elle se scinde en deux et signe à l'envers, Eva Schtued (Schtued, c'est le recours à l'hébraïsme «shtus» qui signifie nonsens), manifestation de dérision dans sa tentative de remonter au temps où l'on parlait l'allemand et le yidich chez elle. Cette mise en abyme par le truchement de l'écriture fait ressortir la destruction de l'allemand dans le récit oral : la narratrice est entrée dans l'ère des métamorphoses. Elle a perdu son identité, mais pas sa souche, le yidich, sa langue maternelle (mameloshn).

17 Il reste son empreinte première, son appartenance à la tradition de ses ancêtres, la marque de sa judéité. En ce sens, le chant yidich contenu dans la lettre fait partie des kinoth (kloglider), chants funèbres relatant les persécutions au cours des siècles. Le livre tout entier s'insère dans la khurbn-literatur, la Trümmerliteratur, la littérature des ruines. La destruction de la Galicie pendant la première guerre mondiale avait vu naître une littérature semblable: An-ski, écrivain et auteur de théâtre yidich (Le dybbouk), a regroupé ces textes littéraires dans une anthologie intitulée Hurbn Galitsie (1914-1917). Ces chants funèbres, même à la première personne, sont l'expression de la mémoire collective : ils se souviennent des morts sans sépulture. Les écrivains juifs galiciens ont tous été marqués par le cataclysme qui a commencé en 1914. On pourrait prétendre que le début de la "Shoah» n'est ni 1933, ni 1939-1945, mais bel et bien 1914. La fin de l'Empire austro-hongrois en est la pierre de touche. Mais pour Eva Deutsch/narratrice, dont la langue de culture était le polonais (la connaissance parfaite de cette langue lui a permis de passer incognito pendant la guerre), le yidich constitue tout au plus un jargon (page 117). Le fait de se couper de sa langue d'origine la fait se couper du « lieu de son véritable ressourcement » qui lui permettrait « la révolte contre la tradition mais aussi (le) jaillissement de vérités cachées ${ }^{24}$ ». De cette " langue de femme », il ne reste que des bribes, des tournures incomplètes, des ébauches de phrases. Il ne s'agit en aucun cas d'un texte en yidich polonais (!) comme le font remarquer les comptes rendus lors de la parution du livre ${ }^{25}$. La langue du récit n'est pas une langue telle qu'on peut l'entendre : elle est issue de la superposition de différents éléments qui peuvent renvoyer à des lieux indistincts dans la conscience personnelle. Il s'agit en quelque sorte d'un sociolecte $^{26}$, mais éclaté, où s'imbriquent l'allemand, l'autrichien et le yidich. Il repose sur un choix délibéré à la fois du témoin et de l'écrivain : en effet, le Je de Die Galizianerin englobe et celui du narrateur et celui du scripteur et celui du lecteur. Il y a d'une part superposition du témoin et de l'écrivain à travers cette non-langue ou cette " interlangue ", d'autre part distance par rapport au discours propre à chacun. C'est sans doute cette distance qui permet à Eva Deutsch de parler : on constate que certains écrivains de la "Shoah» ont utilisé une langue qui n'était pas leur langue maternelle pour pouvoir s'exprimer ${ }^{27}$. La narratrice s'excuse sans cesse d'utiliser une langue qu'elle revendique certes, mais dont elle souligne l'incorrection qui, d'après elle, la fait paraître ridicule («Fehler...Ich spreche Sie an in meiner Sprache. Sie können dabei lachen, es tut nichts», page 49). Cette réflexion est à rapprocher de l'emploi du yidich dans la littérature allemande: couleur locale, dérision antisémite, typisation folklorique ${ }^{28}$. Ici, rien de tel, sinon le refus de part et d'autre d'une langue préétablie, soit dans l'oralité, soit dans l'écriture. Le scripteur, à la recherche du passé, ne peut laisser s'installer une langue dont il aurait pris sur lui de la débarrasser de ses yidichismes, car les rares expressions fonctionnent à la manière de brefs éclairs, introduisant la vie d'autrefois, provoquant un enchevêtrement fulgurant de différentes 
époques qui se chevauchent et dont la chronologie a disparu. Le yidich, souvenir du monde des morts, habite celui des vivants comme des lapsus langagiers. Ainsi la mise en histoire de Je/narrateur repose-t-elle sur la recherche d'une expression propre en même temps qu'elle figure une situation éternellement recommencée: à travers l'oralité se font entendre « les échos de la voix vive ${ }^{29}$ ».

Si le livre constitue un témoignage sur la guerre et sur la manière dont la narratrice, à l'aide de déguisements successifs, parvient à échapper à la mort (elle pourra, munie de faux papiers et bénéficiant de la rare complicité de quelques habitants, se faire passer pour une Polonaise chrétienne), il contient dans le récit oral une infinité de nuances qui permettent un regard diversifié dont le support est la langue. Brigitte Schwaiger, le collecteur et le transcripteur de souvenirs, se glisse dans cette évocation sans avoir l'air de la diriger. Scripteur et narrateur ne peuvent cependant poursuivre le même but: pour le scripteur, il s'agit d'être confronté à ce passé qu'on lui a caché et qui ne peut lui être transmis par un homme (cf. Birer). Pour la narratrice, raconter signifie vivre et faire revivre : mais elle ne sait où va la mener son interlocutrice («Weil ich doch nicht weiß, wohin Sie steuern», page 7). Si son récit est un témoignage sur sa survie, il est plus que cela : il y va de son existence même et de celle des siens qui ont disparu. C'est pourquoi il s'opère dans une langue qui reflète, dans son expression, des événements historiques qui se télescopent dans le temps et qui instaurent une simultanéité déconstructive : ces éléments constitutifs de la langue œuvrent comme un cataclysme. La manière de s'exprimer d'Eva Deutsch apparait comme un parler autre qui rejoint les préocupations de l'écrivain dans l'Autriche d'aujourd'hui : ce pan de littérature orale et écrite constitue un dire qui intègre, en raccourci, les fragments d'une destruction dite par une femme. Ce langage qui relate des événements non pris dans leur chronologie instaure une permanence. C'est tout le contraire d'une mise en histoire qui deviendrait une histoire classée. En utilisant une langue à mi-chemin entre les langues, Eva Deutsch superpose à une langue déstructurée une langue en train de se créer ${ }^{30}$.

\section{NOTES}

1. - Ingeborg Bachmann, Der Fall Franza, Werke 3, Piper § Co. München, 1978, p. 341 sq. (Franza, Actes Sud, 1985).

2. - Brigitte Schwaiger, «Mannsbilder, Frauenzimmer», in Mit einem möcht'ich leben, Wilhelm Heyne Verlag, München, 1987, p. 56 sq. Ces deux expressions - «bonshommes, bonnes femmes » - sont prises au mot (portraits d'hommes, chambres de femmes).

3. - Cf. note 2 .

4. - Wilhelm Reich, La psychologie de masse du fascisme, Payot, Paris, 1974.

5. - Manès Sperber, «Die Wasserträger Gottes», in All das Vergangene, Europaverlag, Wien, 1983

(« Porteurs d'eau », in Ces temps-là, Calmann-Lévy, Paris, 1976).

6. - Joseph Roth, Hiob «Roman eines einfachen Mannes» (1930), Verlag Kiepenheuer § Witsch, Köln, 1982 Job, Valois, Paris, 1931). 
7. - Elisabeth Freundlich, Die Ermordung einer Stadt namens St anislau, «NSVernichtungspolitik in Polen 1939-1945», Österreichischer Bundesverlag, Wien, 1986, L'extermination d'une ville du nom de Stanislaü.

8. - Cf. Charles Baudelaire, "À une Malabaraise ", Euvres complètes, Bibliothèque de la Pléiade, Paris, 1961, p. 156.

9. - Martin Pollack, Nach Galizien, «Von Chassiden, Huzulen, Polen und Ruthenen. Eine imaginäre Reise durch die verschwundene Welt Ostgaliziens und der Bukowina», Ed. Christian Brandstätter, Wien, 1984, En Galicie, « Hassidim, Huzules, Polonais et Rutheniens. Voyage imaginaire à travers le monde disparu de la Galicie orientale et de la Bucovine ».

10. - Christian Ramseyr (Ed.), Im blinden Winkel, «Nachrichten aus Mitteleuropa», Verlag Christian Brandstätter, Wien, 1985. Dans l'angle mort « Nouvelles de la Mitteleuropa ».

11. - Cf. note 5 .

12. - Ruth Beckermann, Die papierene Brücke, Wien, 1985 Le pont de papier.

13. - Galizien als gemeinsame literarische Landschaft, hg.v. Fridum Rinner und Klaus Zerinschek, Innsbrucker Beiträge zur Kulturwissenschaft, Sonderheft 62, Beiträge des 2. Innsbrucker Symposiums polnischer und österreichischer Literaturwissenschaftler, Innsbruck, 1988 La Galicie comme topos littéraire commun, Actes du $2^{\mathrm{e}}$ colloque d'Innsbruck sur la littérature polonaise et autrichienne, $\mathrm{n}^{\circ} 62$.

14. - Op. cit., p. 55.

15. - Cf. Bruno Schulz, «Die mythisierte Wirklichkeit» («La réalité faite mythe »), in Proza, Krakow, 1973, p. 334 sq.

16. - Cf. Hubert Orlowski, «Galizien als Bedrohung. Zum Galizienbild im antisemitischen Lexikon "Sigilla Veri"», in Innsbrucker Beiträge, op. cit., p. 29 sq. (La Galicie comme menace. L'image de la Galicie dans l'Encyclopédie antisémite Sigilla Veri.

17. - Cf. Stefan H. Kaszinski, «Der Tod in Galizien», in Innsbrucker Beiträge, op. cit., p. 29 sq.

18. - Roman Vishniac, Un monde disparu, Seuil, Paris, 1984.

19. - Karl Emil Franzos, «Schiller in Barnow», in Aus Halb-Asien, Erster Band, Verlag von Duncker $\S$ Humblot, Leipzig, 1876, p. 69 sq. («Schiller à Barnov», Images de l’Europe-Asie).

20. - Manès Sperber, op. cit., p. 167.

21. - Paul Celan, «Todesfuge», 1975, in Mohn und Gedächtnis, Stuttgart, 1952 («La fugue de la mort », in Pavot et mémoire).

22. - Klaus Voswinckel à propos de Paul Celan, cité par Harald Weinrich: "Paul Celan ", in Deutsche Literatur der Gegenwart, Bd I. hg.v.Dieter Weber, Alfred Kröner Verlag, Stuttgart, 1976, p. 283.

23. - Lucien Dällenbach, Le récitspéculaire, « Essai sur la mise en abyme », Seuil, Paris, 1977.

24. - Alexandre Derczansky, "Langue et religion: le Yiddish" in Aperçus sur le fait juif, Recherches de science religieuse, tome 66, $\mathrm{n}^{\circ} 4$, Paris, 1978.

25. - Cf. «Rheinische Post» 11.9.82. Düsseldorf, «vorgetragen in dem jüdischen Idiom ihrer polnischen Heimat».

26. - Hans-Peter Althaus: «Soziolekt und Fremdsprache. Das Jiddische als Stilmittel in der deutschen Literatur», in Deutsche Philologie, bd. 100, Sonderheft, Erich Schmidt Verlag, Berlin, 1981.

27. - Nous citerons Elie Wiesel et André Schwarz-Bart.

28. - Cf. Mark H. Gelber, «Das Judendeutsch in der deutschen Literatur», in Juden in der deutschen Literatur, «Ein deutsch-israelisches Symposion, hg.v. Stéphane Moses und Albrecht Schöne, suhrkamp taschenbuch 2063. Frankfurt am Main, 1986 (« Le judéo-allemand dans la littérature allemande ", in Les Juifs dans la littérature allemande).

29. - Paul Zumthor, La lettre et la voix, « De la littérature médiévale », Seuil, Paris, 1987.

30. - Cf. Rokhel Auerbach, Beim letztn weg, farlag isroel-buh, Tel-Aviv, 1977. Ce roman sur le même sujet constitue lui un témoignage en yidich. 


\section{RÉSUMÉS}

Die Galizianerin (La Galicienne) est une œuvre «bicéphale» dont les auteurs sont Brigitte Schwaiger, écrivain, et Eva Deutsch, témoin. Bien que de par sa thématique - la recherche d'une identité féminine - et de par sa problématique - la confrontation avec les événements récents en Autriche (l'antisémitisme et la shoah) - ce livre s'insère dans la production littéraire de l'écrivain, il occupe néanmoins une place à part, due à la langue. Cette langue particulière et qui n'en est pas une, représente une tentative de préserver l'oralité du récit effectué par le témoin à l'intérieur de l'écriture. Le témoignage est scandé par des réponses à des questions non formulées. Cette langue, de l'allemand où perce sans cesse le yidich comme langue originelle, est revendiquée par le narrateur et par le scripteur. Elle se superpose à un univers disparu, la Galicie, qui ne peut plus se dire qu'à travers un langage déstructuré ; elle empêche l'installation d'un dire construit et structuré, d'un dire chronologique sur les événements. Elle devient un condensé d'une histoire personnelle déconstruite qui tente de s'exprimer. Si la langue de la narratrice est l'interstice dans lequel se glisse l'écrivain pour s'essayer à la parole porteuse de sens, les deux opèrent une distance en face d'un mode d'expression qui, dans son étrangeté, permet une parole jamais dite : celle d'une femme prise entre la tradition et la modernité, la culture et la barbarie. Son témoignage constitue une œuvre littéraire, à la fois chant funèbre et sépulture.

Die Galizianerin ist ein «zweiköpfiges» Werk, deren Aurorinnen Brigitte Schwaiger als Schrifstellerin und Eva Deutsch als Zeugin sind. Obwohl dieses Buch auf; die Thematik - Suche nach weiblicher Identität - und die Problematik - Auseinandersetzung mit den jüngsten Ereignissen in Österreich - zurückgreift, nimmt es doch eine Sonderstellung ein, die von der Sprache herrührt. Durch diese eigenartige, an sich nicht vorhandene Sprache wird versucht, das mündliche Erzählen der Zeugin im schriftlichen Erzählen der Schriftstellerin beizubehalten. Oder ist dieses «Unterwegssein zur Sprache» ein fingiertes mündliches Erzählen im literarischen Werk der Autorin? Antworten auf ungeäußerte Fragen bilden die rhythmische Gestaltung der Aussage. Die Sprache - ein mit jiddischen Worten durchsetztes Deutsch -, wird sowohl von der Erzählerin als auch von der Schreiberin beansprucht. Sie fungiert als Abbild einer versunkenen Welt - Galizien -, auf die sich nur noch durch eine entstrukturierte Rede hindeuten läßt; sie verhindert das Einführen eines aufgebauten und gegliederten Aussagens, einer chronologischen Aneinanderreihung von Geschehnissen. Sie wird zum Abriß einer eigenen, abgebauten Geschichte, die immer wieder versucht, zum Ausdruck zu kommen. Ist die Sprache der Erzählerin der Spalt, in den sich die Schreiberin einschleicht, um sich an das bedeutungsvolle Wort zu wagen, so tritt für beide eine Distanz ein, und zwar zu einem Ausdrucksmittel, das in seiner Fremdartigkeit ein noch kaum geäußertes Wort ermöglicht: das Wort einer zwischen Tradition und Modernität, Kultur und Barbarei stehenden Frau. Ihre Aussage bildet ein literarisches Werk, zugleich Klagelied und letztes Geleit.

\section{AUTEUR}

\section{ASTRID STARCK}

Université de Haute Alsace, Mulhouse 